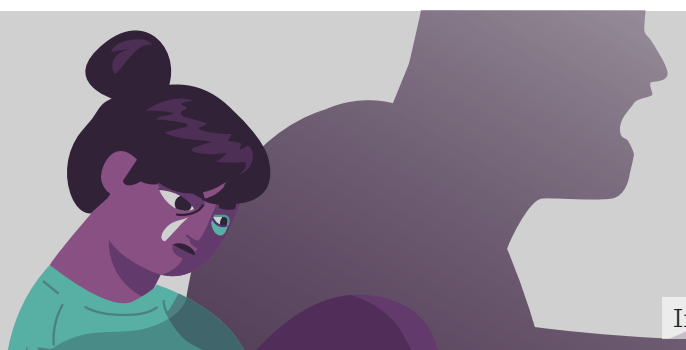

Imágenes de pikisuperstar de freepik

\title{
Concentración espacial de las denuncias por violencia familiar en Ciudad Victoria: un acercamiento mediante la detección de hot spots Spatial concentration of complaints of family violence in Ciudad Victoria: an approach through the detection of hot spots
}

\author{
Víctor Daniel Jurado-Flores*, Francisco Javier Fuentes-Ríos
}

\begin{abstract}
RESUMEN
La violencia familiar es el principal delito cometido a nivel nacional en México. Tamaulipas tiene una tasa de denuncias que se encuentra por encima de la media nacional desde el año 2016 hasta el 2018, destacando el municipio de Victoria con la tasa más alta en ese período. El objetivo de este trabajo fue identificar la concentración espacial de las denuncias por violencia familiar en Ciudad Victoria y especificar la relación entre las desventajas del vecindario y la ubicación en un hot spot (punto caliente). El estudio se basa en la teoría de la desorganización social y tiene como soporte los datos de la Fiscalía General del Estado de Tamaulipas en el periodo 2016-2018. Para ello, se desarrolló el análisis exploratorio de datos espaciales mediante las técnicas de estimación de densidad de kernel, promedio del vecino más cercano, análisis de hot spot $\mathrm{Gi}^{*}$ de Getis-Ord y un análisis confirmatorio mediante una regresión logística binaria, procesamientos desarrollados en el software ArcMap 10.8.1 y en el paquete estadístico R. Los hallazgos indicaron que las denuncias se producen desde un número reducido de unidades geográficas. La prueba del promedio del vecino más cercano mostró que existe agrupamiento estadísticamente significativo $(z=-10.825, P=0.000)$. En el análisis $\mathrm{Gi}^{*}$ de Getis-Ord se detectó que 602 manzanas (9.8\% del total) son hot spots (al $95 \%$ y $99 \%$ de confianza) de alta incidencia, mientras que la regresión logística confirmó que la cantidad de ocupantes por vivienda y los hogares con jefatura femenina están asociados positivamente con la probabilidad de estar dentro de un hot spot de violencia familiar. El patrón de denuncias mostró poca dispersión geográfica y asociación estadística relevante con las variables de desorganización social.
\end{abstract}

PALABRAS CLAVE: violencia familiar, desorganización social, hot spots.

\section{ABSTRACT}

The main crime committed in Mexico is domestic violence. Tamaulipas had a reporting rate above the national average from 2016 to 2018, with Ciudad Victoria having the highest rate during that period. The objective of this work is to identify the spatial concentration of complaints on family violence and to specify the relationship between the neighborhood characteristics and its location in a hot spot. To that aim, the study uses the social disorganization theory and data of complaints taken from the Fiscalia General del Estado de Tamaulipas during the 20162018 period. The exploratory analysis of spatial data was developed by using kernel density estimation techniques, nearest neighbor average, Getis-Ord $\mathrm{Gi}^{*}$ hot spot analysis and confirmatory analysis through binary logistic regression, with the help of the ArcMap 10.8.1 software and $R$ statistical package. The findings indicated that complaints of domestic violence are produced from a small number of geographic units. The nearest neighbor mean test showed that there is a statistically significant grouping of complaints $(z=-10.825, P=0.000)$. In the $\mathrm{Gi}^{*}$ analysis of Getis-Ord, 602 blocks (9.8\% of the total) were identified as hot spots (at $95 \%$ and $99 \%$ confidence), while the logistic regression confirmed that the number of occupants per dwelling and households headed by women are positively associated with the probability of being in a hot spot of family violence. The pattern of the complaints showed little geographic dispersion and relevant statistical associations with the social disorganization variables.

KEYWORDS: domestic violence, social disorganization, hot spots.

*Correspondencia: v.jurado.flores@gmail.com/ Fecha de recepción: 12 de marzo de 2021/Fecha de aceptación: 10 de noviembre de 2021/ Fecha de publicación: 28 de enero de 2022.

El Colegio de Tamaulipas, Coordinación General Académica, Calzada Luis Caballero núm. 1540, Tamatán, Ciudad Victoria, Tamaulipas, México. C. P. 87060. 


\section{INTRODUCCIÓN}

En el año 2018, el Secretariado Ejecutivo del Sistema Nacional de Seguridad Pública (SESNSP) reveló que el total de delitos denunciados en México ascendió a 1989 930, lo que representó una tasa de 1588 por cada 100000 habitantes. Esta cifra tuvo un incremento porcentual del $12.94 \%$ con respecto a 2016. El principal delito en 2018 fue la violencia familiar, con 180187 acusaciones, lo que representó el $9.05 \%$ del total (SESNSP, 2019a).

En 2018, Tamaulipas se ubicaba por encima de la media nacional con una tasa promedio de 196 denuncias de violencia familiar por cada 100000 habitantes, en contraste con la cifra a nivel nacional, que fue de 144. Esta condición se ha mantenido desde 2016 (SESNSP, 2019b), siendo el municipio de Victoria el que ocupa el primer lugar desde 2015, al presentar una tasa promedio de 271 denuncias por cada 100000 habitantes (SESNSP, 2019b).

La violencia familiar es un concepto que engloba varios tipos de actos y de víctimas. El Código Penal de Tamaulipas la define como "el ejercicio de maltrato físico, psicológico, patrimonial o sexual contra cualquier otro miembro de la familia con el que se encuentre o haya estado unido por vínculo matrimonial, de parentesco consanguíneo, afinidad civil" (POE, 2020: 95). La Organización Mundial de la Salud (OMS) la ubica como una de las múltiples formas de violencia interpersonal que engloba acciones contra la pareja y maltratos a niños y ancianos (OMS, 2002).

La violencia familiar es un delito de características especiales, ya que al ocurrir en el ámbito privado es difícil acceder al mismo (Gracia y col., 2015), por ello, los académicos han favorecido la investigación en los determinantes individuales y relacionales sobre los factores explicativos contextuales (Beyer y col., 2015; Gracia y col. 2018; Lila y col., 2019; Hardesty y Ogolsky, 2020; Herrero y col., 2020; Gracia y col., 2021). Aunque en menor escala, dicho fenómeno social también se ha analizado a partir de la influencia de estos últimos fac- tores, abordándolo desde una perspectiva geográfica (Pinchevsky y Wright, 2012; Gracia y col., 2015; 2018; Lila y col., 2019; Gracia y col., 2021). Tomando como marco la teoría de la desorganización social, se ha generado evidencia consistente que vincula una variedad de condiciones negativas del vecindario con un riesgo elevado de ocurrencia de violencia familiar (Gracia y col., 2018; Lila y col., 2019; Gracia y col., 2021).

La teoría de la desorganización social fue desarrollada por académicos de la Escuela de Chicago (Shaw y McKay, 1942). El trabajo de estos autores fue fundamental e implicó un punto de inflexión en el análisis del crimen, ya que se centraron en las características de los vecindarios en lugar de enfocarse en los atributos individuales de los delincuentes.

Existen ciertas características del vecindario que indican desorganización social (nivel económico o educativo bajo, concentración de migrantes, hogares con jefatura femenina y hacinamiento, entre otros), las cuales están asociadas con el incremento del riesgo de casos de violencia doméstica (Pinchevsky y Wright, 2012; Gracia y col., 2015; 2018; 2021). La concentración de población migrante presenta un mayor riesgo relativo de acumulación espacial de dicho suceso (Gracia y col., 2015; Lila y col., 2019). La evidencia derivada de esta forma de abordaje es consistente en distintas ciudades y contextos culturales (Pinchevsky y Wright, 2012; Beyer y col., 2015; Voith, 2017; Gracia y col., 2021).

Las características de desventaja social debilitan los lazos comunitarios y la confianza entre vecinos, reduciendo la capacidad de la comunidad para la acción colectiva y los controles sociales informales del crimen y la violencia, incluida la familiar (Lila y col., 2019; Gracia y col., 2021). Por ello, hay un consenso en la literatura en el sentido de que el riesgo de violencia doméstica está determinado por la interacción de múltiples factores que operan a nivel individual, relacional, comunitario y estructural (Hardesty y Ogolsky, 2020). 
Desde la perspectiva de la eficacia colectiva, las comunidades donde existe mayor comunicación, redes vecinales robustas y confianza, tienden a presentar menores niveles de criminalidad, debido al fortalecimiento de los controles informales (Pinchevsky y Wright, 2012; Browning y col., 2017), los cuales inhiben episodios de violencia familiar ante el efecto del alto capital social de las comunidades (Kirst y col., 2015). En contraparte, los vecindarios que tienen desventajas económicas, inestabilidad residencial y empleo masculino informal son más proclives a presentar este delito (Benson y col., 2003; Gracia y col., 2018; Hernández y col., 2018; Kovacs, 2018; Lila y col., 2019; Gracia y col., 2021).

Los vecindarios caracterizados por tener bajos niveles de ingreso y de educación tienen mayor riesgo relativo de la presencia de esta clase de eventos (Gracia y col., 2021) y otros tipos de violencia y criminalidad (Lila y col., 2019).

Los factores de riesgo no están igualmente distribuidos geográficamente, pero tienden a agruparse. Las diferencias entre áreas residenciales pueden ser mayores que las encontradas entre ciudades o países (Martín-Fernández, 2019; 2020). Por ello, un marco analítico espacial es fundamental para entender el impacto de las desventajas sociales presentes en el vecindario, relacionado con el riesgo de sucesos de violencia familiar (Gracia y col., 2018; 2021). Entonces, el análisis de los factores contextuales, incluidos los comunitarios y estructurales, son clave para entenderla y definir la condición de riesgo de un escenario particular (Kovacs, 2018).

El estudio de la concentración de desventajas sociales a nivel territorial, medidas en formas variadas (Pinchevsky y Wright, 2012), ha generado evidencia robusta para evaluar el grado de amenaza independientemente del análisis de otra clase de factores, sean estos micro o macro sociales (Pinchevsky y Wright, 2012; Voith, 2017).

Es necesario considerar la importancia del lugar y la proximidad territorial para un me- jor entendimiento de la violencia (Anselin y col., 2000; Graif y Sampson, 2009), inclusive la familiar (Caetano y col., 2010; Cunradi y col., 2011; Gracia y col., 2014; Beyer y col., 2015; Gracia y col., 2015). La literatura que aborda el tema de la acumulación de los delitos coincide en que hay zonas específicas de las ciudades en donde la incidencia es alta. Algunas de estas zonas o hot spots son considerados como "crónicos" por la persistencia espacial y temporal de la conducta delictiva (Gorman y col., 2009; Braga y col., 2010; 2012; Weisburd y Amram, 2014; Nogueira y col., 2015; Weisburd, 2015; Weisburd y col., 2017; Favarin, 2018).

A nivel nacional hay una amplia literatura sobre el tema, sin embargo, son pocos los estudios que lo abordan desde una perspectiva del territorio. En Ciudad Juárez, Chihuahua, México se identificaron zonas de alta recopilación de carpetas de investigación abiertas por este hecho delictivo, encontrando colonias de alta prevalencia, tanto en el tiempo como en el espacio (FICOSEC, 2021). En Tamaulipas, Langle (2020) construyó un modelo de factores predictores de violencia hacia la pareja en el hogar, y clasificó a los municipios con mayores probabilidades de presentar este tipo de eventos en función de ciertas características sociodemográficas. Por otra parte, Jurado (2020) examinó en Ciudad Victoria la autocorrelación espacial entre denuncias por violencia familiar y llamadas de emergencia por maltrato infantil y actos violentos hacia la mujer; los resultados indicaron una incidencia diferenciada de estos hechos en pocas colonias de la ciudad.

El objetivo de este trabajo fue analizar el comportamiento espacial de las denuncias por violencia familiar en Ciudad Victoria en el periodo 2016-2018, para determinar la relación entre las variables sociodemográficas y los hot spots, y así identificar la posible asociación de los factores de desorganización social con el riesgo de presentar actos de este delito a nivel de manzana. 


\section{MATERIALES Y MÉTODOS}

\section{Obtención de la población de estudio}

La Fiscalía General del Estado de Tamaulipas (FGETAM) en el contexto de una colaboración interinstitucional, informó que en Ciudad Victoria, Tamaulipas, México, se presentaron 1534 denuncias por violencia familiar en el periodo del 1 de enero de 2016 al 31 de diciembre de 2018. Por razones de confidencialidad, la información solo contenía las coordenadas geográficas de la acusación (latitud y longitud), las cuales se geocodificaron y fueron agregadas a nivel de localidad y de manzana mediante el sistema de información geográfica ArcMap 10.8.1.

\section{Ubicación cartográfica}

La cartografía a nivel de manzana fue obtenida del Censo Nacional de Población y Vivienda 2020 del Instituto Nacional de Geografía y Estadística (INEGI, 2020). Es importante aclarar que el análisis confirmatorio mediante la regresión logística tiene como unidad la manzana y no el hogar, ya que no existen datos ni cartografía oficial que permita desarrollarlo. Los estadísticos descriptivos manejan un total de 6109 unidades geográficas, las cuales responden a los usos habitacionales, y se excluyeron las que no tuvieran usos residenciales (centros comerciales, lotes baldíos, hoteles, comercios, entre otros). La información a nivel de coordenada se geocodificó y cada evento se convirtió en un punto. Para analizar el fenómeno se emplearon técnicas de análisis exploratorio de datos espaciales: densidad de kernel, promedio del vecino más cercano (verifica si los sucesos se encuentran agrupados o dispersos); indicadores locales de autocorrelación espacial mediante el algoritmo Getis-Ord (identificar en qué manzanas se presenta esta agrupación); regresión logística (analiza la relación entre las variables identificadas en la teoría con la probabilidad de que una manzana sea considerada como hot spot).

\section{Densidad de kernel}

La estimación o suavización de kernel es un método para examinar patrones globales de gran escala en datos de puntos. El objetivo de esta técnica es estimar cómo los niveles de eventos varían de manera continua a lo largo de un área de estudio, basada en un patrón de puntos observado para una muestra. La estimación crea un mapa suavizado de valores usando datos espaciales, el cual aparece como un histograma territorial, con el nivel de cada localización a lo largo del mapa reflejando la intensidad del patrón de puntos para los alrededores (Anselin y col., 2000).

La estimación de kernel ha sido aplicada a diferentes campos, particularmente la epidemiología. En el análisis epidemiológico, una distribución de puntos discretos, que representan la incidencia de las enfermedades entre la población, es transformada en un mapa de superficie suavizada continua que indica el riesgo de enfermedad. Al transformar los patrones de puntos espaciales de actos criminales en un mapa suavizado, la estimación de kernel puede ser muy efectiva en la visualización de áreas de criminalidad y riesgo (Anselin y col., 2000).

Desplegar datos de puntos en un mapa puede ser un ejercicio confuso y poco informativo, mientras que una imagen suavizada capta y señala hot spots como áreas de alta densidad que pueden ser verificadas a través del análisis del nivel de significancia estadística, observando si estas zonas de alta densidad están distribuidas de manera aleatoria (Anselin y col., 2000). El estimador kernel de densidad para un conjunto de datos $n$ es definido por:

$$
\hat{f}_{\mathrm{x}}(x)=\frac{1}{N h} \sum_{j=1}^{n} k\left(\frac{x-X_{n}}{h}\right)
$$

Donde $k$ es la sumatoria de jorobas en cada observación, $h$ es el parámetro de suavización, distancia establecida.

\section{Promedio de vecinos más cercanos}

La medida del promedio de vecinos más cercano (ANN, por sus siglas en inglés: Average nearest neighbor) es una herramienta que mide la distancia entre el centroide de un suceso y el de su vecino más cercano y promedia todas las distancias entre ellos. De acuer- 
do con el Environmental Systems Research Institute (ESRI, 2020): "Si la distancia promedio es menor al promedio de una distribución hipotéticamente aleatoria, los puntos se consideran como agrupados. Si la distancia promedio es mayor al promedio de una distribución hipotéticamente aleatoria, los eventos se consideran dispersos". Se calcula dividiendo la distancia promedio observada entre la distancia media esperada. La medida del vecino promedio más cercano es dada por:

$$
A N N=\frac{\bar{D}_{o}}{\bar{D}_{E}}
$$

Donde $\bar{D}_{o}$ es la distancia media observada entre cada evento y su vecino cercano:

$$
\bar{D}_{o}=\frac{\sum_{i=1}^{n} d_{i}}{n}
$$

$\mathrm{YD}_{\mathrm{E}}$ es la distancia media esperada para los eventos dados en un patrón aleatorio:

$$
\bar{D}_{E}=\frac{0.5}{\sqrt{n / A}}
$$

En las ecuaciones anteriores $d_{i}$ equivale a la distancia entre el punto $i$ y el más cercano, $n$ corresponde al total de eventos y $A$ es el área total de estudio. El valor $Z_{A N N}$ es calculado de la siguiente manera:

$$
Z_{A N N}=\frac{\bar{D}_{o}-\bar{D}_{E}}{S E}
$$

Donde:

$$
S E=\frac{0.26136}{\sqrt{n^{2 / A}}}
$$

Si el índice es menor a 1 , el patrón presenta agrupamiento. Si el índice es mayor que 1, la tendencia es hacia la dispersión (ESRI, 2020).

\section{Análisis hot spot con el estadístico $\mathrm{Gi}^{*}$ de Getis Ord}

La prueba $G i^{*}$ de Getis-Ord identifica agrupamientos de puntos que tienen valores más altos que los esperados aleatoriamente. Requiere datos agregados de ubicaciones de puntos de delitos individuales hasta recuentos de estos para áreas pequeñas representadas por puntos centroides. La agregación es requerida porque se espera que haya variaciones en los datos. Si el conteo de casos de un área es alto y los de las zonas vecinas también lo son, la prueba concluye que ambas son parte de un hot spot. Una manzana es una unidad geográfica idónea para la realización de dicha prueba, debido a su nivel de desagregación. Y también es útil esta escala, ya que los procesos de toma de decisiones en seguridad pública precisan de áreas pequeñas (Gorr y col., 2018) para una atención más eficiente.

Este estadístico es aditivo en el sentido que se enfoca en la suma de los valores $j$ en vecindad de $i$. El estadístico $G i^{*}$ permite hacer hipótesis en relación con el agrupamiento. Entonces, el estadístico $G i^{*}$ local es:

$$
G^{*}{ }_{i}=\frac{\sum_{j=1}^{n} w_{i, j} x_{j}-\bar{X} \sum_{J=1}^{n} w_{i, j}}{\frac{\sqrt{\left[n \sum_{j=1}^{n} w_{i, j}^{2}-\left(\sum_{j=1}^{n} w_{i, j}\right)^{2}\right]}}{n-1}}
$$

Donde $x_{i}$ es el valor del atributo para el evento $j$, $w_{i, j}$ es el peso espacial entre el evento $i$ y $j$; n es igual al número total de eventos $\mathrm{y}$ :

$$
\begin{gathered}
\bar{X}=\frac{\sum_{j=1}^{n} x_{j}}{n} \\
S=\sqrt{\frac{\sum_{j=1}^{n} x_{j}^{2}}{n}-(\bar{X})^{2}}
\end{gathered}
$$

El estadístico $G i^{*}$ es un valor $z$, entonces, no se requieren cálculos adicionales. La puntuación $z$ alta y un P-valor pequeño $(<0.05)$ indican agrupamiento de valores altos. Una puntuación $z$ negativa y un $\mathrm{P}$-valor pequeño son evidencia de valores bajos en este aspecto. Entre más alto sea el valor $z$, más intenso será el agrupamiento (ESRI, 2018).

Para hacer el cálculo de los hot spots, primero se obtuvieron los centroides de los polígonos de las unidades geográficas (manzanas), los cuales quedaron expresados en coordenadas geográficas (latitud y longitud). El resultado de este procedimiento es una tabla, en la cual aparecen las coordenadas de cada unidad geográfica y sus respectivas claves de manzana. Las coordenadas obtenidas del cálculo de 
centroide se geocodificaron y el resultado fue una capa de puntos.

El siguiente paso consistió en asignar a cada evento un identificador, que consiste en la clave geográfica de la manzana, con un radio de distancia de $6 \mathrm{~m}$. Este paso permitió hacer una sumatoria de puntos dentro del polígono y entonces asignarle a cada centroide la cantidad de delitos que ocurrieron dentro de la manzana. Por último, se crea una nueva capa en donde la sumatoria de denuncias por manzana se agregó a la capa de puntos, lo que permitió hacer el cálculo de hot spot mediante el estadístico $G i^{*}$ de Getis-Ord.

\section{Regresión logística binomial}

Una vez identificados los hot spots se realizó un análisis confirmatorio mediante una regresión logística binaria. Se clasificaron el total de unidades geográficas (manzanas) con usos habitacionales (6 109) y se identificó si eran o no hot spots. Entonces, se generó una variable dependiente $Y$, que responde a dos categorías $(0$ = no hot spot, 1 = hot spot $)$, entonces $\mathrm{Y}=0,1$; y con esta variable dependiente se desarrolló un modelo de regresión logística binomial con las variables independientes identificadas con la revisión de la literatura.

La regresión logística binomial presenta la siguiente forma:

$\lambda(x)=\ln \left\{\frac{\operatorname{Pr}(Y=1 \mid x)}{\operatorname{Pr}(Y=0 \mid x)}\right\}=\alpha+\left(\beta_{1} x_{1}+\beta_{2} x_{2}+\ldots+\beta_{\mathrm{p}} x_{\mathrm{p}}\right)$

Donde:

$\operatorname{Pr}(Y=1 \mid x)=$ la manzana es hot spot

$\operatorname{Pr}(Y=0 \mid x)=$ la manzana no es hot spot

$\alpha=$ intercepto

$\beta_{1} x_{1}=$ efecto marginal de $x_{1}$

$\beta_{2} x_{2}=$ efecto marginal de $x_{2}$

Las variables independientes que se utilizaron para el modelo de regresión son las siguientes:

\section{Nivel socioeconómico}

- Tasa de desempleo: se calculó mediante la división de la población económicamente acti- va desocupada entre la población económicamente activa (INEGI, 2020).

- Población sin derechohabiencia: se refiere al total de personas que no están afiliadas a servicios médicos en ninguna institución pública o privada (INEGI, 2020). Se utiliza como variable aproximada de la informalidad laboral.

- Viviendas particulares habitadas que disponen de automóvil o camioneta (INEGI, 2020). Se utiliza como variable aproximada del ingreso.

\section{Nivel educativo}

- Grado promedio de escolaridad: es el resultado de dividir el monto de grados escolares aprobados entre las personas mayores de 15 años (INEGI, 2020).

\section{Migración}

- Población de 5 años y más residente en otra entidad en marzo de 2015 (INEGI, 2020).

\section{Características del hogar}

- Hogares con jefatura femenina (INEGI, 2020).

\section{Vivienda}

- Número de personas ocupantes en viviendas particulares habitadas (INEGI, 2020).

\section{RESULTADOS}

La aplicación de las tres pruebas descriptivas arroja resultados que confirman la concentración de las denuncias sobre violencia familiar en Ciudad Victoria. En cuanto a la estimación de densidad de kernel, es importante mencionar que el parámetro crítico para esta prueba es el radio de búsqueda, el cual fue de $300 \mathrm{~m}^{2}$, que permite establecer zonas que no sean muy amplias y que impidan una visión detallada, pero tampoco muy focalizadas y demasiado pequeñas, que son poco prácticas en el análisis.

En la Figura 1 se resaltan los sectores de acumulación de los eventos de acuerdo con la intensidad del color. Las denuncias por violencia familiar tienen un patrón: en primer 




Fuente: desarrollado a partir del mapa cartográfico de INEGI, 2020, incluyendo los datos de incidencia de violencia familiar proporcionados por FGETAM, usando ArcMap 10.8.1.

Figura 1. Estimación de densidad de kernel de denuncias por violencia familiar en la zona de estudio. Figure 1. Kernel density estimation of complaints on family violence in the study zone.

lugar, varias colonias alrededor del centro de la ciudad (San José, Pedro J. Méndez, Morelos, Adolfo López Mateos, entre otras) tienen densidades muy altas de reportes por cada $300 \mathrm{~m}^{2}$. Por otra parte, se identificó otra concentración en el norponiente (Mariano Matamoros, Guadalupe Victoria, Cañón de la Peregrina, Naciones Unidas, entre otras) y surponiente de la ciudad (Luis Echeverría, San Marcos 1 y 2, Estudiantil, entre otras). Por último, un área de menor intensidad en el oriente de la ciudad (América de Juárez, Las Flores y Cuauhtémoc). En la Figura 2 se observan las colonias que presentan mayor acumulación de acusaciones.

Para la prueba del promedio de vecinos más cercanos, en principio se especificó el parámetro del área total de la unidad de análisis
(Ciudad Victoria), la cual fue de $61.322 \mathrm{~km}^{2}$. Establecer este parámetro es un paso importante para la precisión de la prueba. Los resultados del análisis (Tabla 1) apuntan que la distancia media observada (de los casos) a los vecinos cercanos es menor que la distancia media esperada, bajo el supuesto de que las denuncias están distribuidas de manera uniforme y aleatoria. Otro elemento que permite confirmar este patrón es el estadístico $z$, el cual presenta un valor de - 10.825 (muy por debajo de - 2 y - 3, que son considerados valores altamente significativos), lo que implica un alto nivel de significancia estadística del agrupamiento de los eventos. En otras palabras, hay pocas probabilidades de que lo anterior ocurriera al azar, entonces, el fenómeno tiene bases causales. 


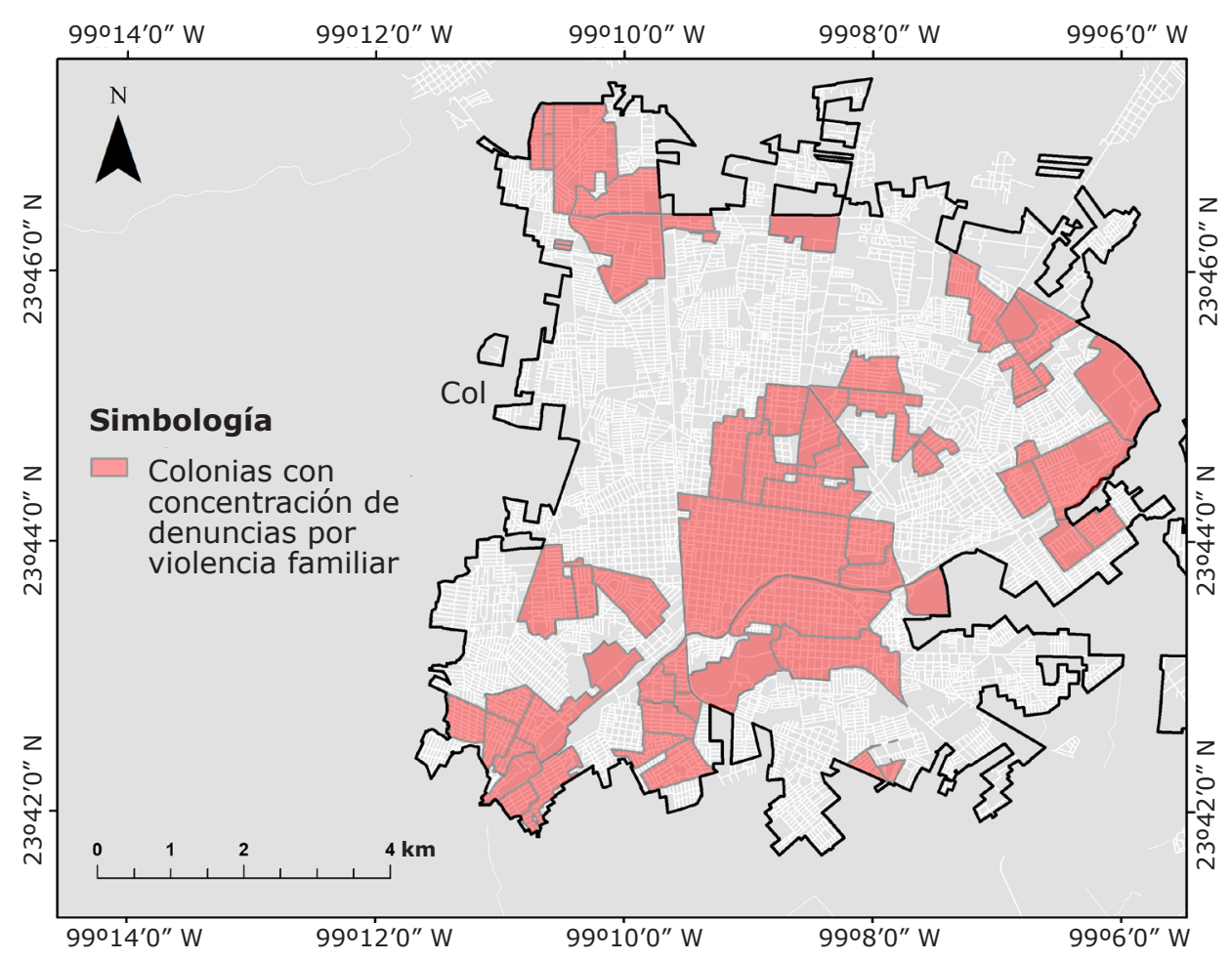

Fuente: modificado a partir de INEGI, 2020 en ArcMap 10.8.1.

Figura 2. Colonias con mayor concentración de denuncias por violencia familiar.

Figure 2. Settlements with greater concentration of complaints of family violence.

Tabla 1. Resumen prueba promedio del vecino más cercano.

Table 1. Summary average nearest neighbor test.

\begin{tabular}{|l|c|}
\hline \multicolumn{1}{|c|}{ Parámetro } & Valor \\
\hline Distancia media observada & $83.797 \mathrm{~m}$ \\
\hline Distancia media esperada & $97.551 \mathrm{~m}$ \\
\hline Índice del vecino más cercano & 0.859 \\
\hline z-score & 10.825992 \\
\hline P-valor & 0.000 \\
\hline
\end{tabular}

La prueba de detección de hot spots de GetisOrd identificó en cuáles manzanas de la ciudad se focalizan unidades geográficas con valores altos de denuncias por violencia familiar rodeadas de unidades con altos valores.

La evidencia del análisis hot spot indica que a nivel de manzana se identifican conglomerados significativos (al $95 \%$ y $99 \%$ de confianza). En la Figura 3 se presentan los resultados de la prueba $G i^{*}$ de Getis-Ord: 602 unidades geográficas (9.8\%), y si solo se conside- ran las unidades con un $99 \%$ de confianza, estas se reducen a 394 (6.4\%) que fueron identificadas como hot spots de alta incidencia, lo que muestra la aglomeración del suceso en un número reducido de unidades geográficas.

En la Tabla 2 se presenta la estadística descriptiva de las variables elegidas para modelar la violencia familiar. Para el aspecto socioeconómico y educativo, a nivel de manzana, el promedio de escolaridad era de $10^{\circ}$ cursados, es decir, al menos un grado cursado en ni- 
vel medio superior. La tasa de desempleo era de 0.16 y la población sin derechohabiencia de 7.14 personas. Mientras que las viviendas que disponían de automóvil fueron 10.5, alcanzando un máximo de 162 automóviles por manzana.

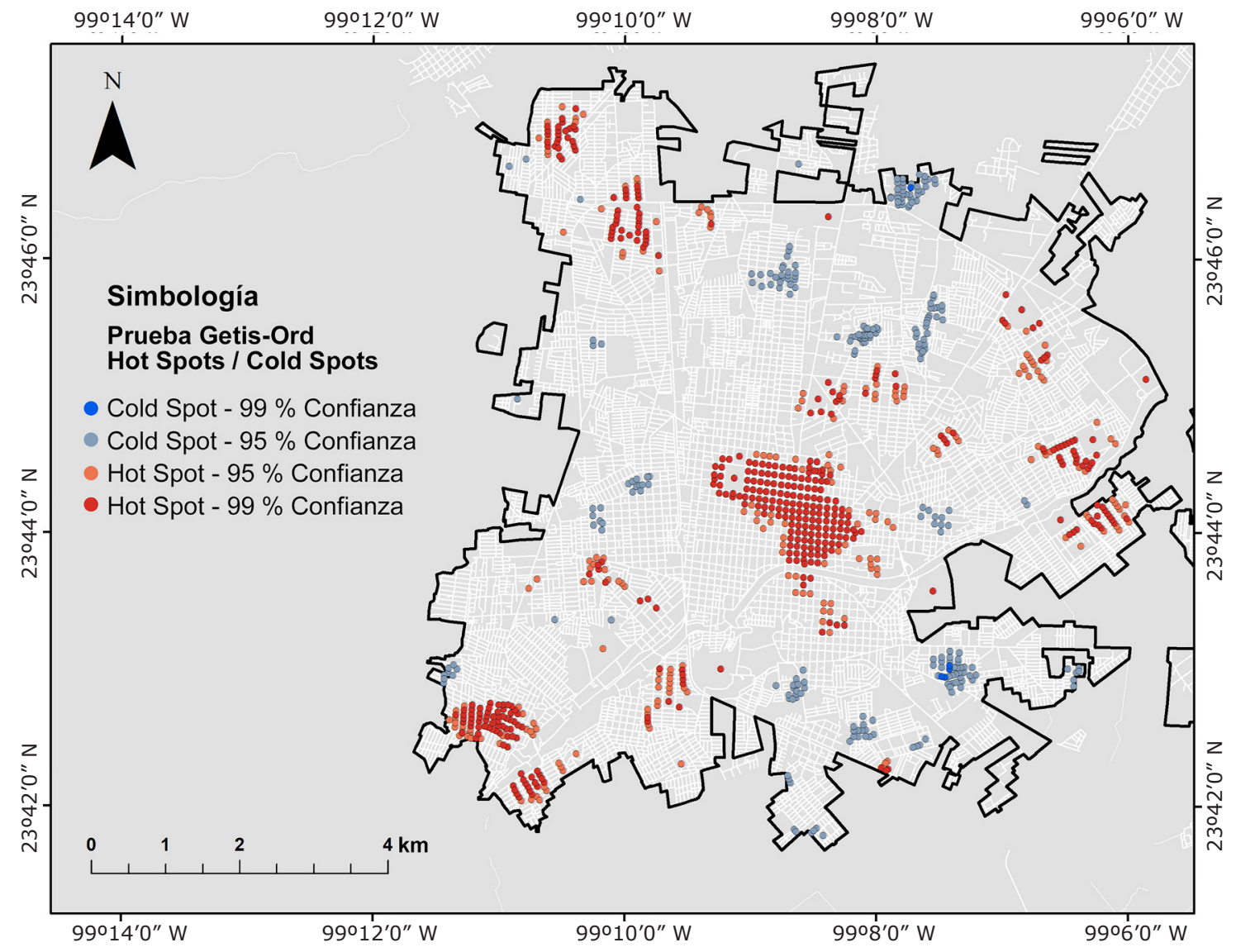

Fuente: modificado a partir de INEGI, 2020 y FGETAM en ArcMap 10.8.1

Figura 3. Análisis de hot spot con $\mathrm{Gi}^{*}$ de Getis-Ord.

Figure 3. Getis-Ord Gi* hot spot analysis.

Tabla 2. Estadísticos descriptivos de las variables de desorganización social por unidad de medida ( $\mathrm{n}=6109$ manzanas).

Table 2. Social Disorganization variables descriptive statistics by measurement unit ( $\mathrm{n}=6109$ blocks).

\begin{tabular}{|l|c|c|c|c|}
\hline \multicolumn{1}{|c|}{ Variable } & Promedio & Desviación & Min & Max \\
\hline Grado promedio de escolaridad & 9.87 & 4.66 & 0 & 19 \\
\hline Hogares con jefatura femenina & 5.22 & 5.59 & 0 & 73 \\
\hline Ocupantes en viviendas particulares habitadas & 53.86 & 48.38 & 0 & 639 \\
\hline Población residente en otra entidad (migración) & 0.48 & 1.99 & 0 & 99 \\
\hline Población sin derechohabiencia & 7.14 & 8.05 & 0 & 97 \\
\hline Tasa de desempleo & 0.16 & 0.36 & 0 & 1 \\
\hline Viviendas particulares habitadas que disponen de & 10.51 & 10.45 & 0 & 162 \\
automóvil o camioneta & 0.1 & 0.3 & 0 & 1 \\
\hline Y= 0,1 (0 = no hot spot, 1 hot spot) & & & 0 \\
\hline
\end{tabular}


Respecto a las características del hogar por manzana, los hogares con jefatura femenina eran 5, y en algunas unidades se tiene la cifra de 73 hogares con esta característica; mientras que los ocupantes por vivienda eran 53.8, con una cifra máxima de 639 personas en la unidad geográfica. La población migrante tenía un promedio de 0.48 personas por manza- na, y en algunas unidades geográficas alcanzaron una cifra de 99 personas migrantes.

Para realizar la prueba de multicolinealidad se construyó la matriz de correlación entre las variables independientes con el objetivo de identificar posibles problemas de multicolinealidad en el modelo de regresión (Tabla 3). Los

Tabla 3. Correlación de las variables de desorganización social.

Table 3. Social disorganization variables correlation.

\begin{tabular}{|c|c|c|c|c|c|c|c|c|}
\hline Variables &  & 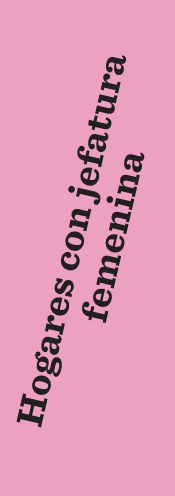 & 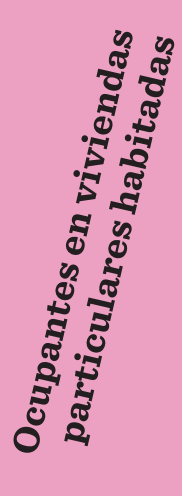 & 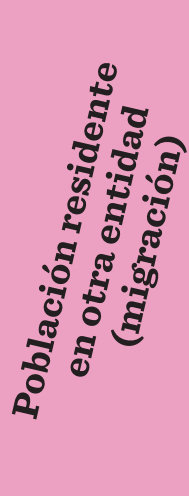 & 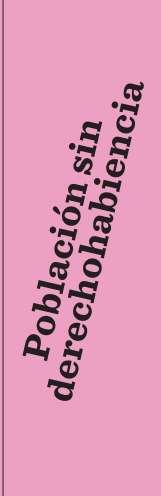 & 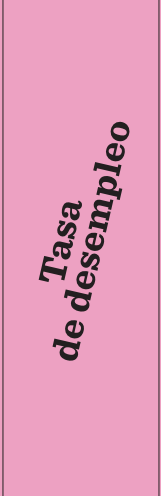 & 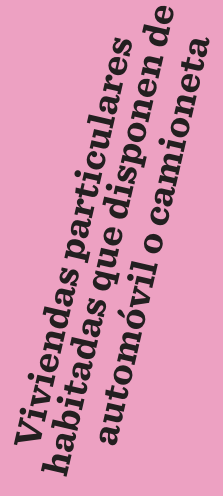 & $\lambda$ \\
\hline $\begin{array}{l}\text { Grado promedio } \\
\text { de escolaridad }\end{array}$ & 1 & & & & & & & \\
\hline $\begin{array}{l}\text { Hogares con } \\
\text { jefatura femenina }\end{array}$ & $0.362^{* * *}$ & 1 & & & & & & \\
\hline $\begin{array}{l}\text { Ocupantes } \\
\text { en viviendas } \\
\text { particulares } \\
\text { habitadas }\end{array}$ & $0.399^{* * *}$ & $0.843^{* * *}$ & 1 & & & & & \\
\hline $\begin{array}{l}\text { Población } \\
\text { residente en } \\
\text { otra entidad } \\
\text { (migración) }\end{array}$ & $0.135^{* * *}$ & $0.254^{* * *}$ & $0.279^{* * *}$ & 1 & & & & \\
\hline $\begin{array}{l}\text { Población sin } \\
\text { derechohabiencia }\end{array}$ & $0.282^{* * *}$ & $0.672^{* * * *}$ & $0.798^{* * * *}$ & $0.212^{* * * *}$ & 1 & & & \\
\hline Tasa de desempleo & $-0.892^{* * *}$ & $-0.390^{* * *}$ & $-0.466^{* * *}$ & $-0.101^{* * *}$ & $-0.369^{* * *}$ & 1 & & \\
\hline $\begin{array}{l}\text { Viviendas } \\
\text { particulares } \\
\text { habitadas que } \\
\text { disponen de } \\
\text { automóvil o } \\
\text { camioneta }\end{array}$ & $0.483^{* * * *}$ & $0.798^{* * *}$ & $0.898^{* * *}$ & $0.301^{* * * *}$ & $0.672^{* * * *}$ & $-0.423^{* * * *}$ & 1 & \\
\hline Y & 0 & $0.076^{* * *}$ & $0.077^{* * * *}$ & 0.005 & $0.088^{* * *}$ & $-0.033^{* *}$ & $0.027^{*}$ & 1 \\
\hline
\end{tabular}

${ }^{*}$ Significativo al $0.05,{ }^{* *}$ Significativo al 0.01, ${ }^{* * *}$ Significativo al 0.001. 
resultados muestran que las siete variables en su mayoría tienen un grado de correlación por debajo del nivel de riesgo de 0.7. Solo se encontraron 5 relaciones entre variables que están por encima de este nivel, por lo que se considera que su número reducido no afecta al desempeño del modelo de regresión.

En el caso del grado promedio de escolaridad tiene una relación negativa y estadísticamente significativa con la tasa de desempleo, lo que indica que conforme incrementa el nivel educativo se reduce la tasa de desempleo.

Los hogares con jefatura femenina tienen una relación positiva y estadísticamente significativa con los ocupantes en viviendas particulares y viviendas que disponen de automóvil. Y el número de ocupantes de viviendas particulares habitadas presenta una relación positiva y estadísticamente significativa con población sin derechohabiencia y viviendas particulares habitadas que disponen de automóvil o camioneta. El resto de las relaciones entre variables no tienen niveles altos de correlación.

Los resultados de la regresión logística destacan que existen asociaciones importantes entre los indicadores derivados de la teoría y las unidades geográficas identificadas como hot spots. Es decir, se interpretan como contribuciones a la probabilidad de que ocurra el evento de interés (en este caso, ser considerado un hot spot). De acuerdo a lo obtenido en el presente trabajo, de las variables seleccionadas para el nivel socioeconómico (Tabla 4), la variable aproximada de ingreso tiene una relación negativa y estadísticamente significativa $(\mathrm{P}<0.001)$ con $\mathrm{Y} 1$ (- 0.055), toda vez que entre mayor sea el ingreso de los habitantes de la manzana (medido de manera indirecta por el número de autos en la vivienda), menor probabilidad de ser considerado como hot spot de violencia familiar. Lo anterior es porque un auto más en la manzana reduce en 5 puntos porcentuales esa probabilidad. Por otra parte, la variable aproximada de la informalidad laboral tiene una relación positiva y estadísticamente significativa $(\mathrm{P}<0.05)$ con $\mathrm{Y} 1$ (0.016), lo que destaca que las condiciones laborales informales aumentan la expectativa de contemplar a la manzana como hot spot, incluso controlando el nivel de ingreso. La variable de tasa de desempleo no es estadísticamente significativa ( $\mathrm{P}>0.05)$, sin embargo, puede deberse a la especificación del modelo; a pesar de ello, las variables de ingreso e informalidad laboral presentan asociación estadística, la primera negativa, y la segunda positiva.

La variable seleccionada para medir la vivienda, evidencia que, acerca de sus ocupantes, existe una relación positiva y estadísticamente significativa con ( $\mathrm{P}<0.01)$ Y1 (0.009); lo que implica que, a mayor población en la manzana, mayor probabilidad de ser catalogado como hot spot. Mientras que la variable de hogares con

Tabla 4. Estimaciones de modelo de regresión logística.

Table 4. Logistic regression estimates.

\begin{tabular}{|l|c|c|c|}
\hline \multicolumn{1}{|c|}{ Parámetro } & Estimación & Error estándar & P-valor \\
\hline (Intercept) & -2.124 & 0.324 & 0.000 \\
\hline Tasa de desempleo & -0.392 & 0.341 & 0.251 \\
\hline Población sin derechohabiencia & 0.016 & 0.008 & 0.045 \\
\hline $\begin{array}{l}\text { Viviendas particulares habitadas que disponen de automóvil } \\
\text { o camioneta }\end{array}$ & -0.055 & 0.012 & 0.000 \\
\hline Ocupantes en viviendas particulares habitadas & 0.009 & 0.002 & 0.001 \\
\hline Grado promedio de escolaridad & -0.029 & 0.027 & 0.294 \\
\hline Población residente en otra entidad (migración) & -0.012 & 0.024 & 0.618 \\
\hline Hogares con jefatura femenina & 0.035 & 0.013 & 0.007 \\
\hline
\end{tabular}


jefatura femenina tiene una relación positiva y estadísticamente significativa $(\mathrm{P}<0.001)$ con Y1 (0.035), esto es, que tienen una mayor probabilidad de estar insertos en manzanas hot spot.

Las variables seleccionadas para medir el nivel educativo, y la migración, no son estadísticamente significativas $(P>0.05$; grado de escolaridad y población residente en otra entidad). De presentarse la primera, su efecto está asociado directamente al ingreso, en donde, a mayor educación, mayor ingreso; en cuanto a la segunda, es necesario considerar el tipo de migración que recibe la ciudad, en términos de nivel educativo y del empleo al que arriban.

\section{DISCUSIÓN}

Los resultados indican que las denuncias por violencia familiar en Ciudad Victoria están espacialmente agrupadas, lo que es consistente con la investigación previa sobre otro tipo de delitos en el marco de la ley de la concentración del crimen (Gorman y col., 2009; Braga y col., 2010; 2011; 2012; Nogueira y col., 2015; Weisburd, 2015; Favarin, 2018; Stanfield y Doherty, 2019), en la cual se hace notar que un reducido porcentaje de unidades geográficas acumulan una gran cantidad de hechos delictivos. La literatura advierte que los factores contextuales tienen influencia en el crimen violento (Braga y col., 2010), crimen hacia la propiedad (Hernández y col., 2018) y otras formas de victimización (Lauritsen, 2001), incluida la violencia familiar (Gracia y col., 2014; Voith, 2017; Stanfield y Doherty, 2019; Kelling y col., 2020).

Desde la perspectiva de la desorganización social, este estudio es consistente con otros que señalan que los vecindarios con bajos niveles de educación y de ingresos tienen mayores probabilidades de presentar sucesos de violencia familiar (Gracia y col., 2015; 2018; 2021). Un entorno con elevados niveles de desventajas económicas e inestabilidad residencial (Benson y col., 2003; Pinchevsky y Wright, 2012; Lila y col., 2019), hombres con empleo informal y comunidades con aislamiento social o desconexión urbana (Pinchevsky y Wright, 2012; Stanfield y Doherty, 2019) son más propensos a presentar estos hechos. Contextos donde hay una alta percepción de problemas comunitarios favorecen la creación de estresores, que propicien la violencia doméstica (Kirst y col., 2015). Las situaciones de desventaja social pueden traducirse en episodios de depresión, ira y estrés emocional (Copp y col., 2015), además de afectaciones negativas en la salud física y mental de la población (Stanfield y Doherty, 2019) y consumo crónico de sustancias psicotrópicas (Weisburd y col., 2017; Carter y col., 2019).

Respecto a la población migrante, donde los resultados no son estadísticamente significativos, es necesario considerar la cualidad y tipo de migración que se analiza. En España tiene bajos niveles educativos y, por consiguiente, también de ingreso (Gracia y col., 2018; Lila y col., 2019; Gracia y col., 2021), mientras que en este estudio la migración tiene otras características tanto educativas como de expectativas de ingreso, así como de inserción laboral.

Los resultados respecto al nivel de ingreso, medido a través de la posesión de auto en el hogar tienen una relación negativa con la agrupación de denuncias, mientras que los hogares con jefatura femenina y la cantidad de ocupantes en relación con el riesgo y/o probabilidad de concentrar eventos de violencia familiar tienen una relación positiva. Ambos resultados son consistentes con lo reportado en ciudades estadounidenses (Pinchevsky y Wright, 2012; Beyer y col., 2015; Voith, 2017) y españolas (Gracia y col., 2015; 2018; Lila y col., 2019; Gracia y col., 2021). Lo anterior, sugiere que existen mecanismos de estos vecindarios que influyen sobre la probabilidad de presentar dicho delito y que estos factores de riesgo están presentes en distintas ciudades y ámbitos culturales. De acuerdo a la teoría de la desorganización social, dichas características de los vecindarios debilitan los lazos sociales y de confianza entre los integrantes de la comunidad, reduciendo la capacidad para la autorregulación y el fortalecimiento de los controles sociales informales.

Los vecindarios socialmente desorganizados pueden estar aislados de los valores y normas 
convencionales respecto al crimen (por ejemplo, desaprobar conductas de violencia familiar), lo que podría conducir a normas y valores que la faciliten, es decir, mayor aprobación y tolerancia de actos violentos, no intervención y cinismo legal (Gracia y col., 2021). Estas normas sociales ad hoc, pueden brindar las bases para un mayor clima social de condescendencia hacia la violencia familiar, por lo que no es señalada, ni condenada como una desviación, y sí considerada como una estrategia permitida en dichos contextos (Gracia y col., 2018).

Por otra parte, los vecindarios que presentan desorganización social pueden convertirse en terrenos fértiles para la socialización de actitudes que acepten la violencia en las relaciones íntimas e internalizarlas como una forma de resolver conflictos. Además, se desestima la importancia de la violencia familiar como un problema social, y con ello se desmovilizan los mecanismos informales de control social (Pinchevsky y Wright, 2012; Gracia y col., 2018; Lila y col., 2019; Gracia y col., 2021).

En términos de política pública, esta clase de análisis es un insumo importante en los procesos de diseño de intervenciones y asignación de recursos públicos para atender dichos eventos. El presente estudio ha identificado que los factores de desventaja de vecindario juegan un rol importante en la reproducción de la violencia familiar y que se concentran en el espa- cio, por lo que dicho conocimiento debe ser incorporado a los esfuerzos de prevención para detectar y reducir el riesgo de este hecho. El problema con la políticas actuales es que se centran en los individuos y se enfocan a víctimas y victimarios o, en el nivel macro, en intervenciones de la educación pública y el reforzamiento de la ley (Gracia y col., 2018). Del presente trabajo se puede enfatizar la importancia de incluir el nivel comunitario (vecindario), apuntando a las áreas de alto riesgo (hot spot) para un enfoque más holístico de la prevención de la violencia familiar.

\section{CONCLUSIONES}

Las denuncias por violencia familiar en el periodo analizado presentan un patrón de agrupamiento espacial. Este hallazgo es importante porque en el contexto latinoamericano pocos estudios lo han abordado desde una perspectiva geográfica a nivel desagregado. La presente investigación ilustra el vínculo entre las desventajas de vecindario y el riesgo de concentrar violencia familiar. Los hallazgos mostrados son un insumo para los tomadores de decisiones en el ámbito de la prevención y atención de este delito para el diseño de políticas públicas, programas y acciones focalizadas, y subrayan la importancia de los esfuerzos de actuación dirigidos a los vecindarios (hot spots) con desventajas sociales, para abocarse a atender y prevenir dicho fenómeno social.

\section{REFERENCIAS}

Anselin, L., Cohen, J., Cook, D., Gorr, W., and Tita, G. (2000). Spatial Analyses of Crime. In D. Duffe (Ed.), Measurement and Analysis of Crime and Justice (pp. 213-262). Estados Unidos: US Department of Justice.

Benson, M., Fox, G., DeMaris, A., and van-Wyk, J. (2003). Neigborhood Disadvantage, Individual Economic Distress and Violence Against Women in Intimate Relationships. Journal of Quantitative Criminology. 19(3): 207-235.

Beyer, K., Baber, A., and Hamberger, K. (2015). Neighborhood Environment and Intimate Partner Violence. Trauma, Violence \& Abuse. 16(1): 16-47.

Braga, A., Hureau, D., and Papachristos, A. (2010). The concentration and stability of gun violence at micro places in Boston, 1980-2008. Journal of Quantitative Criminology. 26:33-53.

Braga, A., Hureau, D., and Papachristos, A. (2011). The relevance of micro places to citywide robbery trends: a longitudinal analysis of robbery incidents at street corners and block faces in Boston. Journal of Research in Crime and Delinquency. 48(1): 7-32.

Braga, A., Papachristos, A., and Hureau, D. (2012). The effects of hot spots policing on crime: an updated systematic review and meta analysis. Justice Quarterly.31(4):633-663. 
Browning, C., Calder, C., Boettner, B., and Smith, A. (2017). Ecological networks and urban crime: the structura of shared routine activity locations and neighborhood-level informal control capacity. Criminology. 55(4): 754-778.

Caetano, R., Ramisetty-Mikler, S., and Harris, R. (2010). Neighborhood Characteristics as Predictors of Male to Female and Female to Male Partner Violence. Journal of Interpersonal Violence. 25(11): 1986-2009.

Carter, J., Mohler, G., and Ray, B. (2019). Spatial Concentration of Opioid Overdose Deaths in Indianapolis: An Application of the Law of Crime Concentration at Place to a Public Health Epidemic. Journal of Contemporary Criminal Justice. 35(2): 161-185.

Copp, J., Kulh, D., Giordano, P., Longmore, M., and Manning, W. (2015). Intimate partner violence in neighborhood context: The roles of structural disadvantage, subjective disorder, and emotional distress. Social Science Research. 53: 59-72.

Cunradi, C., Mair, C., Ponicki, W., and Remer, L. (2011). Alcohol Outlets, Neigborhood Characteristics, and Intimate Partner Violence: Ecological Analysis of a California City. Journal of Urban Health. 88(2): 191-200.

ESRI, Environmental Systems Research Institute (2018). Conjunto de herramientas Asignación de clusters. [En línea]. Disponible en: https://desktop. arcgis.com/es/arcmap/10.3/tools/spatial-statisticstoolbox/hot-spot-analysis.htm. Fecha de consulta: 6 de marzo de 2021.

ESRI, Environmental Systems Research Institute (2020). Cómo funciona vecino más cercano promedio. [En línea]. Disponible en: https://desktop.ar cgis.com/es/arcmap/latest/tools/spatial-statistics-to olbox/h-how-average-nearest-neighbor-distancespatial-st.htm. Fecha de consulta: 6 de marzo de 2021.

Favarin, S. (2018). This must be the place (to commit a crime). Testing the law of crime concentration in Milan, Italy. European Journal of Criminology. 15(6): 702-729.

FICOSEC, Fideicomiso para la Competitividad y Seguridad Ciudadana (2021). Violencia contra las mujeres y niñas: reporte técnico especial. [En línea]. Disponible en: http://observatoriochihuahua.org/wp-con tent/uploads/2021/03/violencia-mujeres-ninas-jrz 2021-otro.pdf. Fecha de consulta: 26 de abril de 2021.

Gorman, D., Zhu, L., and Horel, S. (2009). Drug "hot-spots", alcohol availabity and violence. Drug and
Alcohol Review. 24(6): 507-513.

Gorr, W., Surland, K., and Dodson, Z. (2018). GIS Tutorial for Crime Analysis. New York: Esri Press. 148 Pp.

Gracia, E., López-Quílez, A., Marco, M., and Lila, M. (2018). Neighborhood characteristics and violence behind closed doors: The spatial overlap of child maltreatment and intimate partner violence. PLoS One. 13(6): 1-13.

Gracia, E., López-Quílez, A., Marco, M., Lladosa, S., and Lila, M. (2014). Exploring Neighborhood Influences on Small-Area Variations in Intimate Partner Violence Risk: A Bayesian Random-Effects Modeling Approach. International Journal of Environmental Research and Public Health. 11(1): 866-882.

Gracia, E., López-Quílez, A., Marco, M., Lladosa, S., and Lila, M. (2015). The Spatial Epidemiology of Intimate Partner Violence: Do Neighborhoods Matter? American Journal of Epidemiology. 182(1): 58-66.

Gracia, E., Marco, M., López-Quílez, A., and Lila, M. (2021). Chronic hig risk of intimate partner violence against women in disadvantaged neighborhoods: An eigth year space-time analysis. Preventive Medicine. 148(1): 1-13.

Graif, C. and Sampson, R. (2009). Spatial Hetereogeneity in the Effects of Immigation and Diversity on Neighborhood Homicide Rates. Homicide Studies. 13(3): 242-260.

Hardesty, J. and Ogolsky, B. (2020). A socioecological perspective on intimate partner violence research: a decade in review. Journal of Marriage and Family. 82(2): 454-477.

Hernández, V., Maycotte, E. y Quezada, D. (2018). Geografía del robo a casa habitación en Ciudad Juárez, Chihuahua (2007-2014). Investigaciones Geográficas. 96:1-15.

Herrero, J., Rodríguez-Franco, L., Rejano-Hernández, L., Juarros-Basterretxea, J., and Rodríguez-Díaz, F. (2020). The actor-partner interdependence model in the study of the aggression and victimization within couples: an empirical examination in 361 dyads (2007-2014). Psychosocial Intervention. 29: 165-174.

INEGI, Instituto Nacional de Estadística y Geografía (2020). Censos y Conteos de Población y Vivienda 2020. [En línea]. Disponible en: https://www. inegi.org.mx/programas/ccpv/2020/. Fecha de consulta: 23 de agosto de 2021.

Jurado, V. (2020). Análisis exploratorio de la violencia familiar en Ciudad Victoria 2018, En V. Jurado (Ed.), Contribución al análisis de la violencia fa- 
miliar. [En línea]. Disponible en: http://www.coltam. edu.mx/wp-content/uploads/2020/11/2020-11-12violencia_jurado-1.pdf. Fecha de consulta: 26 de abril de 2021.

Kelling, C., Graif, C., Korkmaz, G., and Haran, M. (2020). Modeling the Social and Spatial Proximity of Crime: Domestic and Sexual Violence Across Neigborhoods, en Journal of Quantitative Criminology. [En línea]. Disponible en: https://link.springer.com/ article/10.1007/s10940-020-09454-w. Fecha de consulta: 15 de febrero de 2021.

Kirst, M., Palma, L.,Zhang, Y., and O'Campo, P. (2015). The Effects of Social Capital and Neighborhood Characteristics on Intimate Partner Violence: A Consideration of Social Resources and Risks. American Journal of Community Psychology. 55(3): 314-325.

Kovacs, R. (2018). The macro-level drivers of intimate partner violence: new evidence from a multilevel dataset. Global Public Health. 13(1): 944-956.

Langle, M. (2020). Análisis de factores predictores de violencia de género dentro del hogar en los municipios del estado de Tamaulipas. En V. Jurado (Ed.), Contribución al análisis de la violencia familiar. [En línea]. Disponible en: http://www.coltam.edu.mx/wp -content/uploads/2020/11/2020-11-12-violencia _jurado-1.pdf. Fecha de consulta: 26 de abril de 2021.

Lauritsen, J. (2001). The Social Ecology of Violent Victimization: Individual and Contextual Effects in the NCVS. Journal of Quantitative Criminology. 17(1): 3-32.

Lila, M., Martín-Fernández, M., Gracia, E., LópezOsorio, J. J., and González, J. (2019). Identifying key predictors of recividism among offenders attending a batterer intervention program: a survival analysis. Psycosocial Intervention. 28(1): 157-167.

Martín-Fernández, M., Gracia, E., and Lila, M. (2019). Psychological intimate partner violence against women in the European Union: a cross-national invariance study. BMC Public Health. 19: 1739.

Martín-Fernández, M., Gracia, E., and Lila, M. (2020). Ensuring the comparability of cross-national survey data on intimate partner violence against women: a cross-sectional, population-based study in the European Union. BMJ Open. 10(1): 1-11.

Nogueira, S., Fonseca, L., and Andresen, M. (2015). Crime concentrations and similarities in spatial crime patterns in a Brazilian context. Applied Geography. 62: 314-324.

OMS, Organización Mundial de la Salud (2002).
Informe mundial sobre la violencia y la salud (sinopsis), Ginebra, Suiza. [En línea]. Disponible en: http:// apps.who.int/iris/bitstream/handle/10665/67411/a7 7102_spa.pdf;jsessionid=3CAB477F269C4209B9754 61E9BBC9F53?sequence=1. Fecha de consulta: 19 de mayo de 2021.

Pinchevsky, G. and Wright, E. (2012). The Impact of Neighborhoods on Intimate Partner Violence and Victimization. Trauma, Violence \& Abuse. 13(2): 112-132.

POE, Periódico Oficial del Estado (2020). Código Penal para el Estado de Tamaulipas, Ciudad Victoria, Tamaulipas. [En línea]. Disponible en: http://po.tama ulipas.gob.mx/wp-content/uploads/2020/08/Codigo_Penal.pdf. Fecha de consulta: 19 de mayo de 2021.

SESNSP, Secretariado Ejecutivo del Sistema Nacional de Seguridad Pública (2019a). Incidencia delictiva del fuero común, nueva metodología, Estatal 2015-2021. [En línea]. Disponible en: https://www. gob.mx/sesnsp/acciones-y-programas/incidenciadelictiva-del-fuero-comun-nueva-metodologia?stte= published. Fecha de consulta: 15 de febrero de 2021.

SESNSP, Secretariado Ejecutivo del Sistema Nacional de Seguridad Pública (2019b). Incidencia delictiva del fuero común, nueva metodología, Municipal 2015-2019. [En línea]. Disponible en: https://www. gob.mx/sesnsp/acciones-y-programas/incidenciadelictiva-del-fuero-comun-nueva-metodologia?sta te=published. Fecha de consulta: 15 de febrero de 2021.

Shaw, C. and McKay, H. (1942). Juvenile Delinquency and Urban Areas, Chicago, University of Chicago Press. $451 \mathrm{Pp}$.

Stanfield, R. and Doherty, E. (2019). Neighborhood Health, Social Structure and Family Violence. Social Science Research. 81: 12-22.

Voith, L. (2017). Understanding the Relation Between Neighborhoods and Intimate Partner Violence: An Integrative Review. Trauma, Violence, \& Abuse. 20(3): 385-397.

Weisburd, D. (2015). The law of concentration and the criminology of place. Criminology. 53(2): 133-15\%.

Weisburd, D. and Amram, S. (2014). The law of concentrations of crime at place: the case of Tel-AvivJaffa. Police Practice and Research. 15(2): 101-114.

Weisburd, D., Shy, M., Amram, S., and Zamir, R. (2017). The relationship between social disorganization and crime at street segments: findings from Tel-Aviv Jaffa census data. In D. Weisburd and J. Eck (Eds.), Unraveling the crime-place connection (pp. 97120). Oxford: Taylor \& Francis. 